\title{
Imbalance in the blood antioxidant system in growth hormone-deficient children before and after 1 year of recombinant growth hormone therapy
}

Maria S Pankratova, Adil A. Baizhumanov, Alexander Yusipovich, Maria Faassen, Tatiana Yu Shiryaeva, Valentina A Peterkova, Svetlana S Kovalenko, Tatiana A Kazakova, Georgy V Maksimov

The aim of our study was to examine the effects of 12-month therapy with recombinant growth hormone $(\mathrm{rGH})$ on the blood antioxidant system in children with growth hormone deficiency (GHD). Total antioxidant capacity (TAC) of plasma was measured by FRAP (ferric reducing antioxidant power or ferric reducing ability of plasma); activities of superoxide dismutase (SOD) and catalase (CAT) in erythrocytes were assessed; non-protein thiols (NT) and ceruloplasmin (CP) levels were also measured. These parameters were determined before and after 12 month of $\mathrm{rGH}$ treatment. Eleven treatment-naive prepubertal children with growth hormone deficiency were included in the study. Another 11 prepubertal children comprised a control group. Before rGH treatment TAC of plasma and NT level in the control group were significantly lower ( $726 \pm 196$ vs. $525 \pm 166 \mu \mathrm{mol} / \mathrm{L}, \mathrm{P}=0.0182$ and $0.92 \pm 0.18$ vs. $0.70 \pm 0.22 \mu \mathrm{mol} / \mathrm{ml}, P=0.0319$, before and after the therapy, respectively). The only parameter that significantly $(19.6 \pm 4.7$ vs $14.5 \pm 3.4$ Units/g $\mathrm{Hb}, \mathrm{P}=0.0396)$ exceeded the same in the control group after rGH therapy was SOD activity. However, none of the measured parameters of antioxidant system in GHD children, except for TAC ( $525 \pm 166$ vs. $658 \pm 115 \mu \mathrm{mol} / \mathrm{L}, P=0.0205$ ), exhibited significant improvement toward the end of the 12-month treatment period, although non-significant changes in CAT activity and CP level were also observed. This work has demonstrated that some parameters of the blood antioxidant system are out of balance and even impaired in GHD children. A 12month treatment with $\mathrm{rGH}$ resulted in a partial improvement of the antioxidant system. 
2 Maria S. Pankratova ${ }^{1}$; Adil A. Baizhumanov²; Alexander I. Yusipovich²; Maria Faassen;

3 Tatyana Yu. Shiryaeva ${ }^{1}$; Valentina A. Peterkova ${ }^{1}$; Svetlana S. Kovalenko ${ }^{2}$; Tatiana A.

4 Kazakova²; Georgy V. Maksimov²

5 'Endocrinology Research Centre, Department of Paediatric Endocrinology, Moscow, Russian

6 Federation

7 2Lomonosov Moscow State University, Biophysics Department, Faculty of Biology, Moscow,

8 Russian Federation

9

10 Corresponding author: Alexander Yusipovich. Mailing address: Leninskie Gory, 24 k1; 119991,

11 Moscow, Russian Federation. Tel: +7(495)9391966. Email sanyavor@gmail.com

12

13 


\section{Introduction}

Free radicals and other reactive species are thought to play an important role in many human diseases. A serious imbalance between production of reactive species and the antioxidant protective system due to increased production of reactive species or low levels of antioxidants leads to oxidative damage (oxidative stress, OS) and development of various disorders (Halliwell, 2004). Therefore, the evaluation of OS could be used as a nonspecific marker of systemic disorders in the human body. Moreover, if the standard treatment shows itself as not sufficient to decrease an ongoing OS, the applied therapy may require modification, for example, by additional administration of vitamins, antioxidants, etc. We believe that some parameters of the blood antioxidant status could be used for evaluation of OS.

It is particularly important to estimate OS in GHD children. Treatment with recombinant growth hormone $(\mathrm{rGH})$ and a subsequent increase in linear growth rate can lead to acceleration of metabolic processes and be accompanied by changes in the blood antioxidant status parameters. Furthermore, an imbalance of the antioxidant parameters was reported in some studies (Evans, 2000; Gonzalez-Duarte, 2012), where patients with adult GHD demonstrated a high degree of OS. Unfortunately, as of today only a small number of publications are dedicated to OS in GHD children, particularly those subjected to treatment with rGH. In fact, we are aware of only one paper (Mohn, 2005). This paper demonstrated that OS parameters (index of susceptibility of low-density lipoprotein (LDL) to in vitro oxidation, malondialdehyde and vitamin E levels) in GHD children were substantially higher than those in the healthy control group, whereas after one year of $\mathrm{rGH}$ therapy these parameters returned to normal levels.

Therefore, in this study we evaluated several parameters of the blood antioxidant system: total antioxidant capacity (TAC) of plasma, activities of superoxide dismutase (SOD) and catalase, non-protein thiols (NT) and ceruloplasmin (CP) levels. These parameters can help to reveal OS in children with GHD and examine the effects of 12-month rGH therapy on the blood antioxidant system.

\section{Materials and Methods}

Eleven treatment-naive GH-deficient patients were included in this study ( 2 girls and 9 boys aged 3 to 9 years). The mean chronological age (CA) was $6.1 \pm 2.2$ years, mean bone age (BA) was $2.6 \pm 0.9$ years. None of them has ever undergone treatment with $\mathrm{rGH}$. 
The parameters of the blood antioxidant system were compared with those in a control group of 11 healthy prepubertal children ( 2 girls and 9 boys; aged 6 to 11 years; mean CA $9.3 \pm 1.4$ years). The control group did not receive any placebo injections.

Standard deviation scores (SDS) for height and growth velocity were calculated using mean and standard deviation of the British reference population as described by Tanner (Tanner, 1966; Tanner, 1976). Pubertal stages are defined accordingly to Tanner (Tanner, 1962).

The SDS values for IGF-1 and IGFBP-3 were calculated for corresponding CA and gender (Lofqvist, 2001; Lofqvist, 2004).

All patients underwent a standard set of clinical and laboratory tests including physical and anthropometric evaluations, x-rays of both hands and wrists, as well as anCT or MRI of the head. To verify the diagnosis, GH-provocation tests were performed: 5 samples were evaluated with clonidine $(0,30,60,90$, and 120 minutes), and 7 samples were evaluated with insulin $(0$, $15,30,45,60,90$, and 120 minutes). A value of less than $10 \mathrm{ng} / \mathrm{ml}$ was an argument in support of the GHD diagnosis (GH Research Society. Consensus guidelines, 2000). Peak values of GH response in both tests were $1.71 \pm 1.45 \mathrm{ng} / \mathrm{ml}$ (with the minimum and maximum values 0.13 and 3.5 accordingly). Idiopathic isolated GH-deficiency was diagnosed in all the cases studied. Clinical and biochemical blood analyses, as well as assays for IGF-1 and IGF binding protein-3 (IGFBP-3) were performed before treatment and after 12 months of rGH treatment. The IGF-1 levels were measured by immunoradiometric assay using a commercial IGF-1 RIA (Nichols Institute Diagnostics, Bad Nauheim); IGFBP-3 level was determined by enzyme immunoassay using commercial DSL-10-6600 ACTIVETM IGFBP3 ELISA kit (DSL, Sinsheim, Germany). Daily rGH injections were subcutaneously administered in the evening. The daily dose of rGH was $0.033 \mathrm{mg}$ per kg body weight (GH Research Society. Consensus guidelines, 2000).

Analyses of the antioxidant system were performed on whole blood samples, which were collected in the fasting state in the morning, before and after 12 months of rGH treatment.

\section{Antioxidant Status Evaluation}

The erythrocyte SOD activity was estimated by inhibition of epinephrine self-oxidation at $25^{\circ} \mathrm{C}$ (Sun, 1978). One unit of SOD activity was defined as that amount of SOD required to cause $50 \%$ inhibition of the oxidation of the epinephrine. The SOD activity was expressed as units per gram of haemoglobin (Units/g Hb). 
75 The level of ceruloplasmin was estimated by measuring the enzymatic reaction with o76 phenylenediamine (Brazhe, 2014).

77 Catalase activity was measured in erythrocytes at $37^{\circ} \mathrm{C}$ according to Aebi (Aebi, 1984). One

78 unit of catalase activity (k) was defined as the amount of enzyme that degrades one mmol $79 \mathrm{H}_{2} \mathrm{O}_{2} / \mathrm{min}$ at initial concentration of $10 \mathrm{mmol} / \mathrm{L}$ at $\mathrm{pH}$ 7.0. The catalase activity was expressed as 80 the unit per gram hemoglobin $(\mathrm{k} / \mathrm{g} \mathrm{Hb})$.

81 Non-protein thiols were analyzed as described by Sedlak et al. (Akhalaya, 2006).

82 The TAC in the blood plasma was estimated by the ferric reducing ability of plasma (FRAP) assay, as described by Benzie and Strain (Benzie, 1996) with modifications: $350 \mu \mathrm{L}$ of distilled water was added to the test tube containing $3 \mathrm{~mL}$ of the reagent (working solution), then $50 \mu \mathrm{L}$ of plasma sample was added and mixed. After 10 minutes the samples were read at a wavelength of $593 \mathrm{~nm}$. The method is based on the reduction of colorless ferric $\left(\mathrm{Fe}^{3+}\right)$ tripyridyltriazine complex in working solution to blue colored ferrous $\left(\mathrm{Fe}^{2+}\right)$ tripyridyltriazine complex at low $\mathrm{pH}$. The TAC values were obtained by comparing the absorption change in the test mixture with those obtained from increasing concentrations of $\mathrm{Fe}^{2+}$ and expressed as $\mu \mathrm{mol}$ 90 of $\mathrm{Fe}^{2+}$ equivalents per $\mathrm{L}$ of sample.

Photometric assay of the blood hemoglobin is based on the transformation of hemoglobin into

92 its haemachrome form by sodium dodecyl sulphate, followed by absorption of the measuring 93 light at $540 \mathrm{~nm}$ (Brazhe, 2014).

Changes in the optical density were recorded with a Hitachi-556 spectrophotometer (Japan).

\section{Statistics}

The results were statistically processed using Statistica software, version 8.0. All data were normally distributed (Kolmogorov-Smirnov test, $\mathrm{p}<0.05$ ). Statistical significance of differences for independent variables, $\mathrm{P}_{1}$ (between parameters of control group and parameters of case group before treatment) and $\mathrm{P}_{2}$ (between parameters of control group and parameters of case 
100 group after treatment), were calculated by one-way ANOVA with the post-hoc Tukey HSD test.

101 The statistical significance of differences for dependent variables, $\mathrm{P}_{3}$ (between parameters of

102 case group before and after rGH treatment) only for case group, was calculated by the paired T-

103 test. Changes were considered significant at $\mathrm{P}<0.05$.

104 The power (one-tailed dependent and one-tailed independent T-test at $\alpha=0.05$ and the sample 105 size of 11) was calculated using the Power Analysis box of Statistica 8.0.

106

\section{Ethics Statement}

The study was approved by the Ethics Committee of the Endocrinology Research Centre, Moscow, Russian Federation (reference number: 14). Written informed consent was obtained from the patients and/or their parents or legal guardians.

\section{Results}

\section{Anthropometric and Biochemical Parameters}

Children treated with rGH demonstrated increases in height, weight, height velocity, height SDS, and height velocity SDS (Table 1). To assess therapeutic efficacy, safety and compliance, IGF-1 SDS and IGFBP-3 SDS were assessed, which showed clear increases during rGH treatment.

Growth parameters after 12 months of rGH therapy significantly exceeded those at baseline (Table 1). After 12 months of treatment the patients' height was significantly increased $\left(96.2 \pm 10.5\right.$ versus $\left.108.0 \pm 10.8 \mathrm{~cm}, \mathrm{P}_{3}=0.000001\right)$, and the increase in growth velocity $(3.4 \pm 1.2$ versus $12.5 \pm 3.5 \mathrm{~cm} /$ years, $\mathrm{P}_{3}=0.000013$ ) suggested that catch-up growth was significant. Thus, catch-up in growth was obvious: height SDS increased from $-3.6 \pm 0.9$ at the onset of therapy and to $-2.2 \pm 1.3$ after 12 months $\left(\mathrm{P}_{3}=0.000030\right)$. Serum IGF-1 SDS level was $-3.1 \pm 1.8$ at baseline and increased to $-0.7 \pm 1.9$ after 12 months of treatment $\left(\mathrm{P}_{3}=0.000689\right)$; IGFBP-3 SDS increased from $-4.0 \pm 3.5$ to $-0.2 \pm 1.7$ after treatment $\left(\mathrm{P}_{3}=0.002364\right)$.

\section{The Antioxidant Status}

To evaluate the blood antioxidant status we selected the most characteristic parameters: total antioxidant capacity (TAC) of plasma measured by FRAP, superoxide dismutase (SOD) and catalase activities, non-protein thiols (NT) and ceruloplasmin levels. The FRAP is proportional to the reducing power of the main non-enzymatic antioxidants in the plasma, 
130 particularly uric and ascorbic acids. This parameter does not reflect the reduced glutathione and 131 liposoluble antioxidants (e.g., vitamin E). Ceruloplasmin and SOD are responsible for both 132 utilization of the superoxide anion radical and the regulation of variable-valence metal levels 133 (copper and iron). Catalase and non-protein thiols play an important role in hydrogen peroxide 134 utilization.

\section{Statistical Power}

The statistical power for all measured parameters was calculated (Table 3). The power varied greatly for different parameters. The highest power was revealed for anthropometric and biochemical parameters (height, weight, growth velocity, height SDS, growth velocity SDS, IGF-1, and IGFBP-3), which exceeded 0.91. For parameters of the blood antioxidant system, statistical power was considerably smaller. In this case, the most profound changes were found for TAC during GH treatment (0.82), and also in comparison of TAC, NT and SOD between the control and experimental groups (0.62, 0.56 and 0.57 , respectively). The lowermost powers were found in changes in SOD and NT in the experimental group during rGH therapy $(0.09$ and 0.25 , respectively) and also for the comparison of catalase and CP between the control and treatment groups ( 0.20 and 0.26 , respectively). 


\section{Discussion}

High power was found for anthropometric and biochemical parameters, in contrast to parameters of the antioxidant system. In general, we revealed small differences in the mean values of antioxidant system parameters and relatively high dispersion (standard deviation), which gives a rather high type 2 error for the small sample volume of 11 persons. In other words, the main drawback in interpretation of the obtained results could be an incapacity to reveal significant differences due to low statistical power, even thought they may be present. This is the reason why it is difficult to confidently reveal the absence of significant changes in parameters like NT, SOD, catalase and CP (power $\leq 0.5$ ). Therefore, we have also considered non-significant differences between the parameters. However, in case of TAC changes during rGH treatment (0.82), and in comparing TAC, NT and SOD between control and experimental groups, the power of the tests was relatively high for a correct interpretation of the obtained results.

We also believe that some discrepancy in the age of patients between control and experimental groups is not crucial for an interpretation of our results because participants of both groups were prepubertal children with similar physiology and similar parameters of the antioxidant system. It is common practice in studies dedicated to the antioxidant status to include prepubertal children aged 2 to 11 years in a single group (see e.g. (Erden-Inal, 2002; Singh, 2002; Llorente-Cantarero, 2013)).

We have demonstrated that parameters of the antioxidant system before treatment are not balanced in GHD children: TAC and NT levels are lower, while SOD activity is elevated albeit statistically non-significantly. The decreased TAC and NT level, the elevated SOD activity and the insignificantly raised level of ceruloplasmin registered in GHD children before rGH treatment, all indicate the presence of OS.

Obviously, a statistically significant resolution of only one parameter (TAC) during treatment indicates an insufficient prevention of the already ongoing OS in GHD children. However, a failure to reveal statically significant differences in TAC and NT (which characterize the total amount of antioxidants in the plasma and blood, respectively) after treatment between the groups, and also a non-significant decline in $\mathrm{CP}$ level (increase in $\mathrm{CP}$ level indicates inflammatory processes) still may suggest an improvement of the antioxidation state in children after therapy. 
In our work we observed some evidence of blood antioxidant system improvements after $\mathrm{rGH}$ treatment, which is supported by the absence of a significant difference in TAC and NT after treatment between the control and treatment groups (using one-way ANOVA with post-hoc Tukey HSD test, $\mathrm{p}<0.05$ ). Moreover, antioxidaton parameters such as TAC, NT and CP (but not SOD and catalase) came back to normal levels during treatment. Although these changes are small (not reaching statistical significance), we can conclude that these findings in general agree with data reported in Mohn et al. (Mohn, 2005), who showed an improvement in the antioxidant status, evidenced by a decline in free radicals in GHD children after rGH treatment.

\section{References}

2000. Consensus guidelines for the diagnosis and treatment of growth hormone (GH) deficiency in childhood and adolescence: summary statement of the GH Research Society. GH Research Society. The Journal of Clinical Endocrinology and Metabolism, 85:3990-3993. DOI: 10.1210/jcem.85.11.6984/.

Aebi H. 1984. Catalase in vitro. Methods in Enzymology, 105:121-126. DOI: 10.1016/s00766879(84)05016-3.

Akhalaya MY, Platonov AG, Baizhumanov AA. 2006. Short-term cold exposure improves antioxidant status and general resistance of animals. Bulletin of Experimental Biology and Medicine, 141:26-29. DOI: 10.1007/s10517-006-0084-5.

Benzie IFF, Strain JJ. 1996. The Ferric Reducing Ability of Plasma (FRAP) as a Measure of "Antioxidant Power": The FRAP Assay. Analytical Biochemistry, 239:70-76. DOI: 10.1006/abio.1996.0292.

Brazhe NA, Baizhumanov AA, Parshina EYu, Yusipovich AI, Akhalaya MY, Yarlykova Y, Labetskaya OI, Ivanova SM, Morukov BV, Maksimov GV. 2014. Studies of the blood antioxidant system and oxygen-transporting properties of human erythrocytes during 105-day isolation. Human Physiology, 40:804-809. DOI: 10.1134/s0362119714070020.

de Oliveira EP, Burini RC. 2012. High plasma uric acid concentration: causes and consequences. Diabetology \& Metabolic Syndrome, 4:12. DOI: 10.1186/1758-5996-4-12.

Duplancic D, Kukoc-Modun L, Modun D, Radic N. 2011. Simple and rapid method for the determination of uric acid-independent antioxidant capacity. Molecules, 16:7058-7068. DOI: 10.3390/molecules16087058.

Evans LM, Davies JS, Anderson RA, Ellis GR, Jackson SK, Lewis MJ, Frenneaux MP, Rees A, Scanlon MF. 2000. The effect of GH replacement therapy on endothelial function and oxidative 
stress in adult growth hormone deficiency. European Journal of Endocrinology, 142:254-262. DOI: $10.1530 /$ eje.0.1420254.

Gonzalez-Duarte D, Madrazo-Atutxa A, Soto-Moreno A, Leal-Cerro A. 2012. Measurement of oxidative stress and endothelial dysfunction in patients with hypopituitarism and severe deficiency adult growth hormone deficiency. Pituitary, 15:589-597. DOI: 10.1007/s11102-011$0374-4$.

Halliwell B, Whiteman M. 2004. Measuring reactive species and oxidative damage in vivo and in cell culture: how should you do it and what do the results mean? British Journal of Pharmacology, 142:231-255. DOI: 10.1038/sj.bjp.0705776.

Lofqvist C, Andersson E, Gelander L, Rosberg S, Blum WF, Wikland KA. 2001. Reference Values for IGF-I throughout Childhood and Adolescence: A Model that Accounts Simultaneously for the Effect of Gender, Age, and Puberty. The Journal of Clinical Endocrinology and Metabolism, 86(12): 5870-5876. DOI: 10.1210/jcem.86.12.8117.

Lofqvist C, Andersson E, Gelander L, Rosberg S, Blum WF, and Wikland KA. 2004. Binding Protein-3 (IGFBP-3) and the Ratio IGF-I/IGFBP-3 throughout Childhood and Adolescence. The Journal of Clinical Endocrinology and Metabolism 12(2): 1587-1596. DOI: 10.1210/jc.20040812 .

Mohn A, Marzio D, Giannini C, Capanna R, Marcovecchio M, Chiarelli F. 2005. Alterations in the oxidant-antioxidant status in prepubertal children with growth hormone deficiency: effect of growth hormone replacement therapy. Clinical endocrinology (Oxford), 63:537-542. DOI: 10.1111/j.1365-2265.2005.02378.x.

Sun M, Zigman S. 1978. An improved spectrophotometric assay for superoxide dismutase based on epinephrine autoxidation. Analytical Biochemistry, 90:81-89. DOI: 10.1016/00032697(78)90010-6.

Tanner, JM 1962. Growth at Adolescence: With a General Consideration of the Effects of Hereditary and Environmental Factors Upon Growth and Maturation from Birth to Maturity. Oxford: Blackwell Scientific Publications.

Tanner JM, Whitehouse RH, Takaishi M. 1966. Standards from birth to maturity for height, height velocity and weight velocity: British children, 1965. Part II. Archives of Disease in Childhood, 41: 613-635. DOI: 10.1136/adc.41.220.613.

Tanner JM, Whitehouse RH. 1976. Clinical longitudinal standard for height, weight, height velocity, weight velocity and stages of puberty. Archives of Disease in Childhood, 51: 170-179. DOI: 10.1136/adc.51.3.170. 


\section{1}

Figure 1: Changes in TAC (a), NT level (b), erythrocyte SOD (c) and catalase (d) activities, CP level (e) after a 12 month rGH treatment (case-control data).

The data presented as a standard box and whisker plot (where the length of the box is an interquartile range) with the mean (closed square) and experimental data (open circle), the ends of the whiskers represent the maximal and minimal values. In the picture data of the control group (black box) and that of the case group before (red box) and after (blue box) $\mathrm{rGH}$ treatment is shown. $P_{1}$ is the statistical significance between parameters of the control group and those of the case group before treatment; $P_{2}$ is the statistical significance between parameters of the control group and those of the of case group after treatment. The experimental data were evaluated by the one-way ANOVA with post-hoc Tukey HSD test. The significant difference $(P<0.05)$ for $P_{1}$ and $P_{2}$ is shown by black rectangles. 

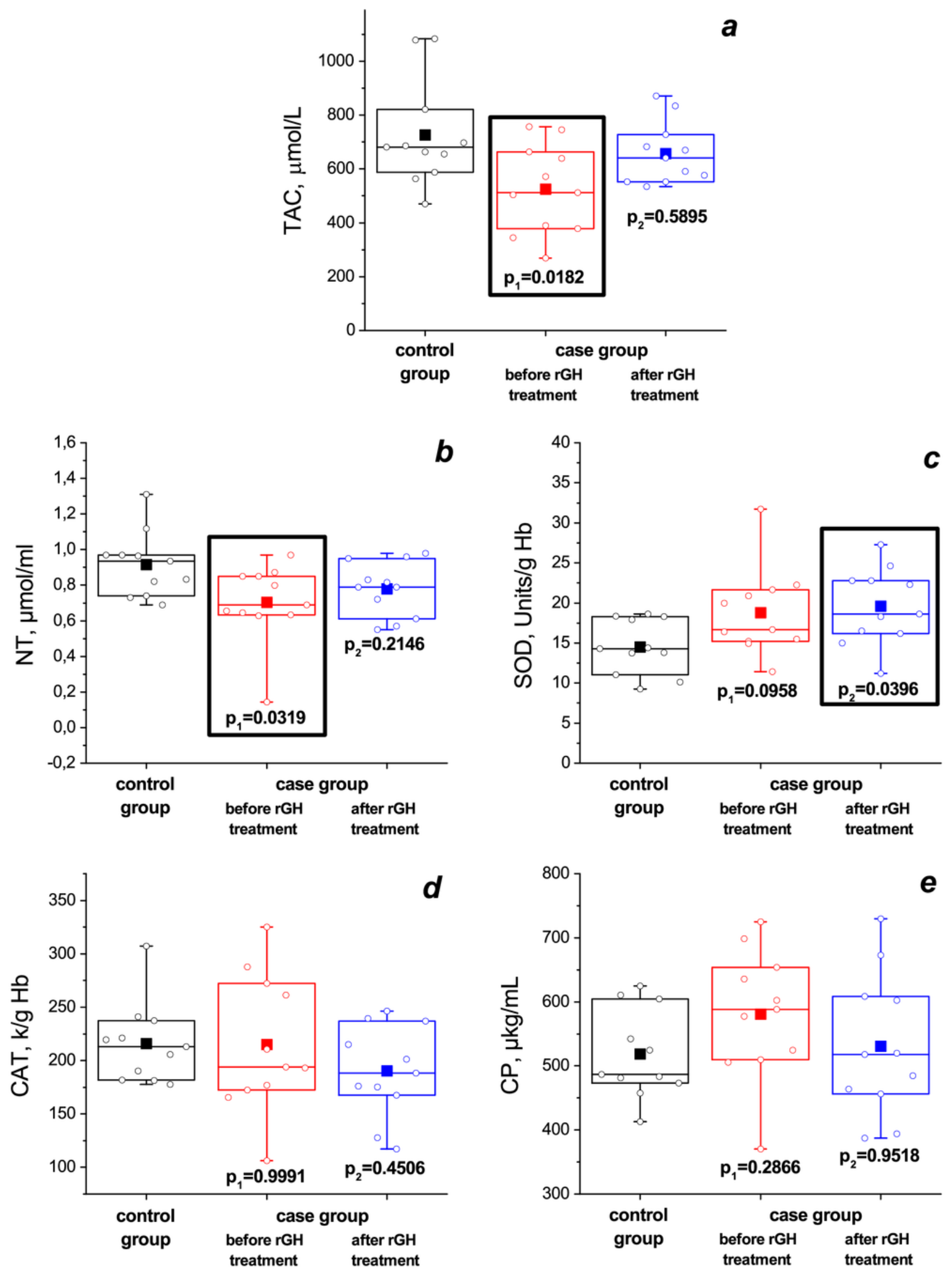


\section{2}

Figure 2: Changes in TAC (a), NT level (b), erythrocyte SOD (c) and catalase (d) activities, CP level (e) during the 12 month rGH treatment (longitudinal data).

The data of case group before and after rGH treatment is shown in picture. Different symbols corresponds to the parameters obtained in various patients before and after the treatment (data of each patient is connected by line). The mean values \pm standard deviations for case group before and after treatment is shown as open red circles with whiskers. $P_{3}$ is the statistical significance between parameters of case group before and after $\mathrm{rGH}$ treatment. The experimental parameters were evaluated by the paired T-test, $\mathrm{p}<0.05$. Significant difference between data is marked by an asterisk. 

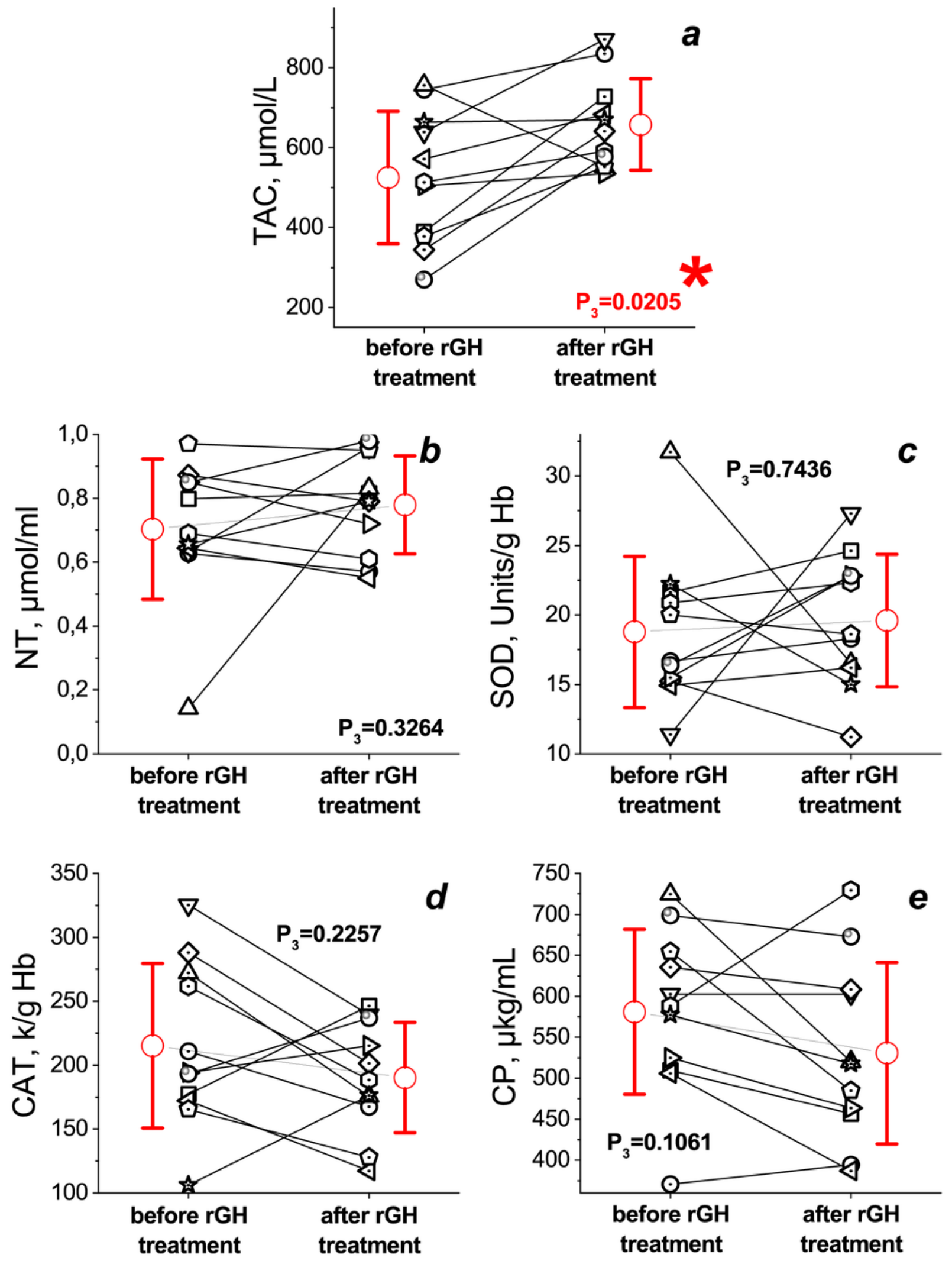
Table $\mathbf{1}$ (on next page)

Table 1: Effect of growth hormone therapy on anthropometric and biochemical parameters of GHD children. 


\begin{tabular}{|c|c|c|}
\hline Parameters & Before GH therapy & After 12 months of GH therapy \\
\hline Height, cm & $96.2 \pm 10.5$ & $\begin{array}{c}108.0 \pm 10.8 \\
\mathrm{P}_{3}=0.000001 *\end{array}$ \\
\hline Weight, kg & $15.2 \pm 3.9$ & $\begin{array}{c}17.8 \pm 5.5 \\
\mathrm{P}_{3}=0.001118^{*}\end{array}$ \\
\hline Growth velocity, $\mathrm{cm} /$ years & $3.4 \pm 1.2$ & $\begin{array}{c}12.5 \pm 3.5 \\
\mathrm{P}_{3}=0.000013 *\end{array}$ \\
\hline Height SDS & $-3.6 \pm 0.9$ & $\begin{array}{c}-2.2 \pm 1.3 \\
\mathrm{P}_{3}=0.000030 *\end{array}$ \\
\hline Growth velocity SDS & $-3.2 \pm 1.8$ & $\begin{array}{c}7.4 \pm 3.9 \\
\mathrm{P}_{3}=0,000026^{*}\end{array}$ \\
\hline IGF-1 SDS & $-3.1 \pm 1.8$ & $\begin{array}{c}-0.7 \pm 1.9 \\
\mathrm{P}_{3}=0.000689 *\end{array}$ \\
\hline IGFBP-3 SDS & $-4.0 \pm 3.5$ & $\begin{array}{c}-0.2 \pm 1.7 \\
\mathrm{P}_{3}=0.002364 *\end{array}$ \\
\hline
\end{tabular}

$\overline{\mathrm{P}_{3} \text { indicates the statistical significance between parameters of the case group before and after }}$ rGH treatment, using the paired T-test, $\mathrm{P}<0.05$ (significant difference between data is marked by an asterisk). 
Table 2 (on next page)

Table 2: Effect of growth hormone therapy on parameters of the blood antioxidant status of GHD children. 


\begin{tabular}{|c|c|c|c|}
\hline Parameters & Control & Before GH therapy & $\begin{array}{c}\text { After } 12 \text { months of GH } \\
\text { therapy }\end{array}$ \\
\hline $\begin{array}{l}\text { Total antioxidant capacity of } \\
\text { plasma, } \mu \mathrm{mol} / \mathrm{L}\end{array}$ & $726 \pm 196$ & $\begin{array}{c}525 \pm 166 \\
\mathrm{P}_{1}=0.0182 *\end{array}$ & $\begin{array}{c}658 \pm 115 \\
\mathrm{P}_{2}=0.5895 \\
\mathrm{P}_{3}=0.0205^{*}\end{array}$ \\
\hline Non-Protein Thiols, $\mu \mathrm{mol} / \mathrm{ml}$ & $0.92 \pm 0.18$ & $\begin{array}{c}0.70 \pm 0.22 \\
\mathrm{P}_{1}=0.0319^{*}\end{array}$ & $\begin{array}{l}0.78 \pm 0.15 \\
\mathrm{P}_{2}=0.2146 \\
\mathrm{P}_{3}=0.3264\end{array}$ \\
\hline $\begin{array}{l}\text { Superoxide Dismutase } \\
\text { (SOD), Units/g Hb }\end{array}$ & $14.5 \pm 3.4$ & $\begin{array}{c}18.8 \pm 5.4 \\
\mathrm{P}_{1}=0.0958\end{array}$ & $\begin{array}{c}19.6 \pm 4.7 \\
\mathrm{P}_{2}=0.0396^{*} \\
\mathrm{P}_{3}=0.7436\end{array}$ \\
\hline Catalase, $\mathrm{k} / \mathrm{g} \mathrm{Hb}$ & $216 \pm 38$ & $\begin{array}{c}215 \pm 64 \\
\mathrm{P}_{1}=0.9991\end{array}$ & $\begin{array}{c}190 \pm 43 \\
\mathrm{P}_{2}=0.4506 \\
\mathrm{P}_{3}=0.2257\end{array}$ \\
\hline Ceruloplasmin, $\mu \mathrm{g} / \mathrm{mL}$ & $518 \pm 70$ & $\begin{array}{c}581 \pm 100 \\
P_{1}=0.2866\end{array}$ & $\begin{array}{c}531 \pm 111 \\
\mathrm{P}_{2}=0.9518 \\
\mathrm{P}_{3}=0.1061\end{array}$ \\
\hline
\end{tabular}

$\mathrm{P}_{1}$ indicates the statistical significance between parameters of the control group and those of the case group before treatment, $\mathrm{P}_{2}$ is the statistical significance between parameters of the control group and those of the case group after treatment; values were evaluated using the one-way ANOVA with post-hoc Tukey HSD test, $\mathrm{p}<0.05 . \mathrm{P}_{3}$ is the statistical significance between parameters of case group before and after $\mathrm{rGH}$ treatment, using the paired T-test, $\mathrm{p}<0.05$. Significant differences between data are marked by an asterisk. 


\section{Table 3 (on next page)}

Table 3: The calculated values of statistic power. 


\begin{tabular}{l|c}
\hline Parameter & Power \\
\hline
\end{tabular}

Dependent data (one-tailed dependent T-test $\alpha=0.05$, the sample size - 11)

\begin{tabular}{l|c}
\hline Height, $\mathrm{cm}$ & 1.00 \\
Weight, $\mathrm{kg}$ & 0.99 \\
Growth velocity & 1.00 \\
Height SDS & 1.00 \\
Growth velocity SDS & 1.00 \\
IGF-1, nMol/L & 0.92 \\
IGFBP-3, nMol/L & 1.00 \\
IGF-1 SDS & 1.00 \\
IGFBP-3 SDS & 0.98 \\
Total antioxidant capacity of plasma, $\mu \mathrm{mol} / \mathrm{L}$ & 0.82 \\
Non-Protein Thiols, $\mu \mathrm{mol} / \mathrm{ml}$ & 0.25 \\
Superoxide Dismutase (SOD), Units/g Hb & 0.09 \\
Catalase, $\mathrm{k} / \mathrm{g} \mathrm{Hb}$ & 0.33 \\
Ceruloplasmin, $\mu \mathrm{g} / \mathrm{mL}$ & 0.50 \\
\hline
\end{tabular}

Independent data (one-way ANOVA $\alpha=0.05$, the sample size - 11)

\begin{tabular}{l|l}
\hline Total antioxidant capacity of plasma, $\mu \mathrm{mol} / \mathrm{L}$ & 0.62
\end{tabular}

Non-Protein Thiols, $\mu \mathrm{mol} / \mathrm{ml} \quad 0.56$

Superoxide Dismutase (SOD), Units/g Hb $\quad 0.57$

Catalase, $\mathrm{k} / \mathrm{g} \mathrm{Hb} \quad 0.20$

\begin{tabular}{r|r} 
Ceruloplasmin, $\mu \mathrm{g} / \mathrm{mL}$ & 0.26 \\
\hline
\end{tabular} 